\title{
Infecção de cães domésticos (Canis lupus familiaris, Linnaeus, 1758) da região metropolitana de Guaratuba, Paraná, Brasil por microfilárias circulantes de Acanthocheilonema reconditum (Grassi, 1899)
}

\section{Infection in domestic dogs (Canis lupus familiaris, Linnaeus, 1758) from Guaratuba, Paraná, Brazil by circulating microfilariae of Acanthocheilonema reconditum (Grassi, 1889)}

\author{
Luiz Carlos Leite ${ }^{1 *}$; Ennio Luz ${ }^{2}$; Silvana Maris Cirio ${ }^{3}$; Marcelle Cirio Leite ${ }^{4}$; \\ Hermínio de Paula Molinari ${ }^{5}$; Ana Carolina Bazo Zadorosnei ${ }^{5}$; \\ Tricia Maria Ferreira de Souza Oliveira ${ }^{6}$; Selene Cirio Leite ${ }^{7}$
}

\section{Resumo}

\begin{abstract}
Os quadros epidemiológicos das zoonoses periodicamente sofrem modificações em alguns de seus aspectos, envolvendo a emergência ou reemergência de agravos à saúde do homem e dos animais. Neste cenário sujeito a rápidas transformações, a análise da prevalência de doenças, a exemplo das filarioses diagnosticadas em cães no litoral do Estado do Paraná, apresenta significada relevância. O presente estudo foi realizado com 422 cães e teve como objetivo determinar a prevalência de microfilárias circulantes de Acanthocheilonema reconditum tanto nos animais alojados no canil municipal, como naqueles domiciliados no bairro COHAPAR II, ambos localizados na região metropolitana da cidade de Guaratuba-Paraná, através de dois exames hematológicos: avaliação do sangue a fresco pela técnica da gota espessa e método de Knott modificado por Newton e Wright. Trinta dos animais avaliados $(7,10 \%)$, 22 machos e oito fêmeas, foram positivos para microfilárias de $A$. reconditum.
\end{abstract}

Palavras-chave: Microfilárias, infecção natural, zoonoses

\begin{abstract}
The epidemiologic frameworks of zoonosis periodically change in some of its aspects, involving the emergence or reemergence of men and animals' worsening of health. In this scenario, in which rapid changes can happen, the analysis of the prevalence of diseases, as the filariasis diagnosed in dogs from the coastline of Paraná, has significant relevance. This study has been carried out with 422 dogs and aimed to determine the prevalence of circulating microfilariae of Acanthocheilonema reconditum, both
\end{abstract}

1 Prof. do Curso de Medicina Veterinária, Universidade Estadual do Centro-Oeste, UNICENTRO, PR, Guarapuava, PR. E-mail: lcmouraleite@gmail.com

2 Prof. Sênior, Universidade Federal do Paraná, UFPR Curitiba, PR. E-mail: ennioluz@hotmail.com.br

3 Prof a do Curso de Med. Veterinária, Faculdade Evangélica do Paraná, FEPAR, Curitiba, PR. E-mail: silvana.cirio@gmail.com

4 Discente, Curso de Bacharelado em Ciências Biológicas Pontifícia Universidade Católica do Paraná, PUCPR, Curitiba, PR. E-mail: mar_leite92@hotmail.com

5 Médico Veterinário, Prefeitura Municipal de Guaratuba, Guaratuba, PR. E-mail: herminiomolinari@gmail.com

Médica Veterinária- Autônoma, Curitiba, PR. E-mail:anacarolinavet@bol.com.br

Prof ${ }^{a}$ do Curso de Medicina Veterinária, Universidade de São Paulo, USP, Pirassununga, SP. E-mail:triciaoliveira@yahoo.com.br

7 Médica Veterinária, Mestranda Curso de Ciências Biológicas, Universidade Federal do Paraná, UFPR, Curitiba, PR. E-mail: seleneleite@gmail.com

* Autor para correspondência 
in animals accommodated at the municipal kennels, as those that live in the district COHAPAR II, both places located in the metropolitan area of the city of Guaratuba-Paraná, through two blood tests: evaluation of fresh blood, by the technique of the 'thick drop' and method of Knott modified by Newton and Wright. Thirty of the evaluated animals (7.10\%), twenty two males and eight females, showed the presence of microfilariae of $A$. reconditum as result.

Key words: Microfilariae, natural infection, zoonosis

Os nematóides filarideos são caracterizados por apresentarem tropismo nos tecidos de seus hospedeiros e pela dependência de artrópodos hematófagos como vetores. Entre as espécies comumente encontradas infectando cães destacam-se Dirofilaria repens, Dirofilaria immitis, Acanthocheilonema dracunculoides, Acanthocheilonema reconditum, Brugia ceylonensis, Brugia pahangi e Brugia malayi (RANI et al., 2010).

Acanthocheilonema reconditum é um helminto responsável por filariose em cães, encontrado nos tecidos subcutâneo eperi-renal.Após oacasalamento, as fêmeas fecundadas originam microfilárias, que podem ser encontradas principalmente na circulação sanguínea periférica desta espécie animal. Como hospedeiros intermediários e vetores destacamse as pulgas das espécies Ctenocephalides canis, Ctenocephalides felis felis e Pulex irritans e os malófagos Trichodectes canis e Heterodoxus spiniger (TORRES; FIGUEIREDO, 2007). A transmissão da larva infectante aos cães ocorre através das picadas de pulgas ou de malófagos no momento do repasto sanguíneo (LINARDI, 2004). $\mathrm{O}$ diagnóstico fundamenta-se no encontro de microfilárias no sangue. A técnica de gota espessa (KNIGHT, 1977) examina o sangue a fresco para detectar a presença e avaliar padrões de tamanho e movimentação de microfilárias, diferenciando as de Acantocheilonema reconditum, que realizam movimentos erráticos e deslocam-se com rapidez, enquanto as de Dirofilaria immitis, outro filarídeo parasita de cães, são dotadas de movimentos ondulantes serpentiformes e movem-se lentamente (BRITO et al., 2001). Tanto microfilárias de $D$. immitis como de $A$. reconditum podem ser encontradas na circulação sanguinea de cães infectados, daí a importância de sua diferenciação. As microfilárias de $A$. reconditum medem aproximadamente $250,0 \pm 8,0 \mu \mathrm{m}$ de comprimento e 4,5 $\pm 1,2 \mu \mathrm{m}$ de largura com um corpo redondo e curvado. Os machos adultos medem 13,0 mm de comprimento e as fêmeas 17,0-32,0 mm (HUYNH; THEAN; MAINI, 2001). As de D. immitis são maiores, medindo cerca de 298,1 $\pm 5,5 \mu \mathrm{m}$ de comprimento e 7,3 $\pm 0,3 \mu \mathrm{m}$ de largura, enquanto os machos adultos medem $12,0 / 20,0 \mathrm{~cm}$ e as fêmeas $25,0 / 30,0 \mathrm{~cm}$ de comprimento. Normalmente a infecção por $A$. reconditum destaca-se por apresentar caráter transitório e sem conseqüências patológicas (KNIGHT, 1987), sendo diagnosticada na maioria das vezes de forma acidental. Em algumas ocasiões, os animais afetados manifestam elevadas eosinofilia e contagem leucocitária. Estudos realizados por Hashem e Badawy (2008) comprovaram presença de anemia hemolítica acompanhada de distúrbios hepáticos e das funções renais em 14 cães parasitados por $A$. reconditum. Entretanto, a maioria das filarias parasitas de cães apresenta comprovado potencial para infectar seres humanos, podendo dar origem a quadros patológicos (HUYNH; THEAN; MAINI, 2001; FOROULIS et al., 2005; SABU; DEVADA; SUBRAMANIAN, 2005; RANI et al., 2010).

No Brasil ainda é subestimado o percentual de cães microfilarêmicos e/ou portadores de formas adultas de Acanthocheilonema reconditum, motivado pela escassa quantidade de publicações existentes a respeito do assunto. No estado da Bahia, Barros, Santos e Almeida (1993) avaliaram 500 amostras de sangue de cães, registrando oito $(1,6 \%)$ infectadas por microfilárias. Idêntica amostragem examinada em Cuiabá-MS, por Fernandes et al. (1999) comprovou contaminação em três $(0,6 \%)$. Amostras sanguíneas de 611 cães em Recife-PE 
avaliadas pelo teste de ELISA e técnica de Knott (1939) modificada por Newton e Wright (1956) identificou $42(6,9 \%)$ animais microfilarêmicos (ALVES et al., 1999). Um inquérito hemoscópico em 1519 cães domiciliados em Maceió-AL, reportou $15(1,3 \%)$ portadores de microfilárias (BRITO et al., 2001).

Amostras do sangue de 256 cães distribuídos em onze municípios da área litorânea do estado do Paraná, avaliadas por Reifur, Thomaz-Soccol e Montiani-Ferreira (2004) mediante visualização direta (gota espessa), técnica de Knott modificada e teste ELISA revelaram a presença de microfilárias de $A$. reconditum em $58(22,6 \%)$ animais.

Também em outros países foram registrados achados do parasita. Em Hyderabad-Paquistão, mediante uso da técnica de Knott modificada, Gadahi et al. (2008) detectaram quatro cães infectados (1,33\%) entre 200 examinados. Através da mesma técnica Ranjbar-Bahadori e Hekmatkah (2007) em Garmsar, Iran, diagnosticaram dois cães microfilarêmicos (1,64\%), entre 122 animais examinados. Em Taiwan, Wang (1997) utilizando processo semelhante, examinou amostras de sangue e soro de 180 cães de rua, encontrando 22 (12,2\%) animais portadores de microfilárias. $\mathrm{Na}$ Itália, Cringoli et al. (2001) avaliaram 351 amostras de sangue de cães assintomáticos utilizando a mesma técnica, demonstrando microfilaremia em 56 amostras positivas (15,9\%). Em Istambul-Turquia, Toparlak et al. (2005) analisaram 286 amostras de sangue de cães, obtendo 2 amostras positivas $(0,69 \%)$. Estudos realizados na Índia por Rani et al. (2010) reportaram achados de microfilárias de $A$. reconditum em 12 (9,3\%) de 139 cães microfilarêmicos.

A ocorrência de microfilárias e formas adultas de $A$. reconditum parasitando seres humanos foi descrita nos Estados Unidos por Greene, Otto e Greenough III (1978) que diagnosticaram achados de microfilárias similares morfologicamente a $A$. reconditum em uma paciente de 12 anos de idade, portadora de Lupus Eritematoso Sistêmico, a qual apresentou microfilaremia durante exacerbação da doença. No mesmo país uma fêmea de Acantocheilonema spp. foi removida da câmara anterior do olho direito de um homem com 32 anos de idade (BEAVER et al., 1980). Ainda Beaver (1989) reportaram mais três casos de filariose intraocular causada por Acanthocheilonema spp com remoção dos parasitas.Na Austrália Huynh, Thean e Maini (2001) descreveram o encontro de uma fêmea adulta de $A$. reconditum com $32 \mathrm{~mm}$ de comprimento, retirada viva e intacta do espaço subconjuntival do olho direito de um paciente com 62 anos de idade.

Em decorrência de registros no litoral do Estado do Paraná (REIFUR; ThOMAZ-SOCCOL; MONTIANI-FERREIRA, 2004), vários outros aspectos devem ser investigados periodicamente, para a correta avaliação da ocorrência de nematóides filarídeos, tanto em cães errantes quanto nos domiciliados.

O presente estudo teve por objetivo analisar a prevalência de $A$. reconditum a partir de amostragem representativa de cães oriundos da área metropolitana da cidade de Guaratuba, estado do Paraná.

O trabalho foi desenvolvido no município de Guaratuba - PR, localizado em área litorânea do estado do Paraná, a $119 \mathrm{Km}$ de Curitiba, em uma planície peninsular arenosa, com uma das frentes na baia de Guaratuba a Noroeste, e outra frente no oceano Atlântico a Sudeste. Possui $22 \mathrm{~km}$ de praias, altitude de $15 \mathrm{~m}$, Latitude $-25^{\circ} 53^{\prime} 60^{\prime \prime} \mathrm{S}$ e Longitude $-48^{\circ} 34^{\prime} 0^{\prime \prime} \mathrm{W}$. Sua área é de 1.328,480 $\mathrm{km}^{2}$ e a população total de 32.088 habitantes, com 28.793 indivíduos componentes da população urbana residente (IBGE, 2010).

Amostras de sangue de 422 cães procedentes do Canil Municipal e do bairro COHAPAR II em Guaratuba, Paraná foram analisadas. Após o exame clínico prévio de todos os animais, foram coletados $5,0 \mathrm{ml}$ de sangue em frascos contendo anticoagulante 
EDTA (ácido etileno diamino tetracético a 10,0\%), por punção direta da veia jugular. As amostras foram acondicionadas em caixas de isopor com gelo e enviadas ao Laboratório PET IMAGEM Diagnósticos Veterinários em Curitiba-Pr, onde foram armazenadas em refrigerador à temperatura de $2-4^{\circ} \mathrm{C}$, processadas e examinadas no prazo máximo de 24 horas após a coleta. Na identificação de $A$. reconditum foram utilizados o exame direto do sangue a fresco e o teste de Knott (1939) modificado por Newton e Wright (1956). Após a realização dos testes, procedeu-se a avaliação microscópica em objetiva de 4x e 10x para a realização da micrometria das microfilárias. Foram identificadas microfilárias de $A$. reconditum medindo entre 249-252 $\mu \mathrm{m}$ de comprimento e 4,4- 4,6 $\mu \mathrm{m}$ de largura, com o corpo apresentando gancho cefálico característico, a cauda curvada em forma de gancho e movimentando-se de forma rápida e errática. Através das mesmas técnicas utilizadas, microfilárias de Dirofilaria immitis foram igualmente identificadas em dois animais: o primeiro, de aproximadamente seis anos de idade reagiu de forma positiva nos três testes laboratoriais utilizados. O segundo cão, com quatro anos de idade acusou positividade apenas no Snap Test. As amostras de sangue foram avaliadas mediante visualização direta, micrometria das microfilárias, Teste de Knott modificado por Newton e Wright e Snap Test (LEITE et al., 2007). Animais sem raça definida (SRD) predominaram na amostragem obtida $(93,3 \%)$ de 422 cães procedentes do canil municipal e do bairro COHAPAR II, ambos situados na periferia do município de Guaratuba, estado do Paraná. Trinta amostras $(7,10 \%)$ apresentaram resultados positivos para $A$. reconditum no teste de Knott modificado por Newton e Wright (22 machos e oito fêmeas). Na visualização direta do sangue (gota espessa) dos mesmos animais, amostras de vinte e um machos e sete fêmeas continham microfilárias.

Foram analisadas 213 amostras (101 fêmeas e 112 machos) procedentes do canil municipal. Vinte e dois animais albergavam microfilárias de $A$. reconditum quinze machos e sete fêmeas (Tabela 1 ).
A amostragem sanguínea de 209 cães (81 fêmeas e 128 machos) no bairro COHAPAR II detectou microfilaremia por $A$. reconditum em oito animais, sete machos e uma fêmea (Tabela 1).

O percentual de amostras contendo microfilárias de $A$. reconditum reportado no presente trabalho de pesquisa $(7,10 \%)$ é inferior aos achados de Reifur, Thomaz-Soccol e Montiani-Ferreira (2004) no litoral do Paraná (22,6\%), semelhante aos obtidos por Alves et al. (1999) em Recife-PE (6,9\%) e superior aos registrados por Barros, Santos e Almeida (1993) no estado da Bahia (1,6\%), Brito et al. (2001) em Maceió-AL (1,3\%), Fernandes et al. (1999) em Cuiabá-MS (0,6\%) e igualmente inferior aos obtidos por Cringoli et al. (2001) na Itália (15,9\%), Wang (1997) em Taiwan (12,2\%), Rani et al. (2010) na Índia (9,3\%), e superior aos relatados por Zahler, Glaser e Gothe (1997) na Alemanha (3,7\%), Uche e Odunze (1988) na Nigéria (2,5\%), Ranjbar-Bahadori e Hekmatkah (2007) no Iran (1,33\%), Gadahi et al. (2008) no Paquistão (1,33\%) e Toparlak et al. (2005) na Turquia, (0,69\%). Em relação à presença de microfilaremia, comprovada através do teste de gota espessa, ocorreu percentual maior em cães $(4,97 \%)$ que o encontrado por Brito et al. (2001) em Maceió-AL (2,6\%). As fêmeas apresentaram grau de infecção ligeiramente superior $(1,65 \%)$ ao reportado no mesmo trabalho $(1,3 \%)$.

Amostras avaliadas pelo teste de Knott modificado por Newton e Wright em machos $(5,2 \%)$ demonstraram microfilárias em porcentagem superior a obtida por Alves et al. (1999) na cidade de Recife-PE (4,7\%). Entretanto em fêmeas houve menor percentual $(1,8 \%)$ quando comparado com registro do autor na mesma pesquisa $(2,1 \%)$. Uma das razões do encontro de maior percentual de microfilárias em machos pode estar relacionada com sua utilização à noite na guarda das áreas peridomiciliares das residências, estando desta maneira mais expostos ao ataque de mosquitos hematófagos; normalmente durante este mesmo período as fêmeas ficam protegidas no interior dos domicilios, diminuindo o grau de exposição. 
Apesar do considerável percentual de cães infectados oriundos da área de investigação, os casos de infecção por $A$. reconditum diagnosticados nesta pesquisa não foram considerados como autóctones, uma vez que era desconhecida a procedência dos animais alojados no canil municipal de Guaratuba.

Tabela 1. Características fenotípicas e idade dos cães positivos para Acanthocheilonema reconditum detectados no Canil municipal de Guaratuba (1 a 22) e Bairro COHAPAR II (23 a 30) em Guaratuba (PR).

\begin{tabular}{|c|c|c|c|c|c|c|c|}
\hline CÃES & IDADE & SEXO & PORTE & RAÇA / PELAGEM & DATA COL. & VD & KNOTT \\
\hline 1 & $4 \mathrm{~A}$ & $\mathrm{M}$ & PEQ & POODLE / PRETO & $29 / 05 / 2001$ & + & + \\
\hline 2 & $3 \mathrm{~A} 5 \mathrm{M}$ & $\mathrm{F}$ & PEQ & SRD / PRETO E BEGE & $29 / 05 / 2001$ & + & + \\
\hline 3 & $4 \mathrm{~A} 6 \mathrm{M}$ & M & PEQ & SRD / CASTANHA & $29 / 05 / 2001$ & + & + \\
\hline 4 & $5 \mathrm{~A} 6 \mathrm{M}$ & M & MÉD & SRD/BRANCO E PRETO & $29 / 05 / 2001$ & + & + \\
\hline 5 & $2 \mathrm{~A} 9 \mathrm{M}$ & M & PEQ & SRD / CASTANHA & $29 / 05 / 2001$ & + & + \\
\hline 6 & $7 \mathrm{~A}$ & $\mathrm{~F}$ & MÉD & SRD/BRANCO E PRETO & $02 / 07 / 2001$ & + & + \\
\hline 7 & $3 \mathrm{~A}$ & M & GRA & SRD / BEGE & $01 / 08 / 2001$ & + & + \\
\hline 8 & $2 \mathrm{~A} 6 \mathrm{M}$ & M & GRA & SRD/BRANCO E PRETO & $13 / 09 / 2001$ & + & + \\
\hline 9 & 3 A $3 \mathrm{M}$ & M & MED & SRD / PRETO E BEGE & $20 / 09 / 2001$ & + & + \\
\hline 10 & $4 \mathrm{~A} 6 \mathrm{M}$ & $\mathrm{F}$ & MÉD & SRD/BRANCO E PRETO & $20 / 09 / 2001$ & + & + \\
\hline 11 & $3 \mathrm{~A}$ & M & PEQ & SRD / TRICOLOR & $20 / 09 / 2001$ & + & + \\
\hline 12 & 4 A $3 \mathrm{M}$ & $\mathrm{F}$ & MÉD & SRD / PRETO E BEGE & $20 / 09 / 2001$ & + & + \\
\hline 13 & $3 \mathrm{~A} 4 \mathrm{M}$ & $\mathrm{F}$ & GRA & ROTTWEILLR / PADRÃO & $20 / 09 / 2001$ & + & + \\
\hline 14 & $2 \mathrm{~A} 6 \mathrm{M}$ & M & MÉD & SRD / CINZA E PRETO & $27 / 09 / 2001$ & + & + \\
\hline 15 & 6 A $2 \mathrm{M}$ & M & MÉD & SRD / BEGE E BRANCO & $18 / 10 / 2001$ & + & + \\
\hline 16 & $4 \mathrm{~A}$ & M & GRA & SRD/BRANCO E PRETO & $18 / 10 / 2001$ & + & + \\
\hline 17 & 2 A $3 \mathrm{M}$ & M & PEQ & SRD / PRETO E BEGE & $21 / 01 / 2002$ & - & + \\
\hline 18 & $4 \mathrm{~A}$ & $\mathrm{~F}$ & MÉD & SRD / CASTANHA & $21 / 02 / 2002$ & - & + \\
\hline 19 & $1 \mathrm{~A} 6 \mathrm{M}$ & M & PEQ & SRD / PRETA E CANELA & $20 / 04 / 2002$ & + & + \\
\hline 20 & $2 \mathrm{~A} 6 \mathrm{M}$ & M & MÉD & SRD / TRICOLOR & $11 / 05 / 2002$ & + & + \\
\hline 21 & $5 \mathrm{~A} 6 \mathrm{M}$ & M & PEQ & SRD / DOURADO & $20 / 07 / 2002$ & + & + \\
\hline 22 & $2 \mathrm{~A}$ & $\mathrm{~F}$ & GRA & SRD/PRETO E MARROM & $20 / 08 / 2002$ & + & + \\
\hline 23 & $2 \mathrm{~A}$ & M & PEQ & SRD/BRANCO E PRETO & $11 / 05 / 2002$ & + & + \\
\hline 24 & $4 \mathrm{~A}$ & M & PEQ & SRD / BRANCA & $15 / 02 / 2003$ & + & + \\
\hline 25 & $4 \mathrm{~A}$ & M & MÉD & SRD / DOURADA & $07 / 05 / 2003$ & + & + \\
\hline 26 & $2 \mathrm{~A}$ & M & PEQ & $\mathrm{SRD} / \mathrm{BEGE}$ & $09 / 01 / 2004$ & + & + \\
\hline 27 & $5 \mathrm{~A}$ & M & PEQ & SRD / TRICOLOR & $15 / 01 / 2004$ & + & + \\
\hline 28 & $4 \mathrm{~A}$ & M & PEQ & SRD/BRANCO E PRETO & $15 / 01 / 2004$ & + & + \\
\hline 29 & $3 \mathrm{~A}$ & M & GRA & SRD / BRANCO & $15 / 01 / 2004$ & + & + \\
\hline 30 & $3 \mathrm{~A}$ & $\mathrm{~F}$ & PEQ & SRD/BRANCO E PRETO & $15 / 01 / 2004$ & + & + \\
\hline
\end{tabular}

SRD. - Sem Raça definida V.D. - Teste de Visualização Direta (Gota Espessa) KNOTT - Teste de Knott modificado, M - Macho, F - Fêmea, A - Anos, M - Meses, PEQ - pequeno, MÉD - médio, GRA - grande.

Fonte: Elaboração dos autores. 
No presente trabalho, observou-se a presença de Acanthocheilonema reconditum em 30 cães $(7,10 \%)$ de 422 que foram examinados na cidade de Guaratuba, estado do Paraná. Os animais estudados não apresentaram sintomatologia típica, exceto microfilaremia. Os resultados obtidos confirmam a existência do filarídeo parasitando cães no litoral do Paraná. Devido ao risco potencial de transmissão, a que estão expostas as pessoas que tem contato direto com animais de estimação, outros estudos serão necessários para avaliar a correlação entre fatores ambientais, socioeconômicos, condições de saneamento básico, freqüência de outras parasitoses e a sensibilização da comunidade para o problema. Outras medidas incluem a realização de sorologia em cães suspeitos ou recém-adquiridos para evitar aquisição de animais portadores assintomáticos.

O controle das pulgas e malófagos, hospedeiros intermediários de nematóides filarídeos, também deve ser realizado periodicamente, utilizando-se produtos inseticidas de comprovada eficácia, por aplicação direta no animal. A presença comprovada de animais microfilarêmicos, em locais que se destacam por condições de temperatura e umidade, adequadas ao crescimento e proliferação de artrópodos vetores, associada à precariedade das condições de saneamento básico, indicam cenário que deve receber atenção especial. Fornecer condições básicas de infraestrutura, para pessoas e animais domiciliados sob permanente risco de agravos à saúde é essencial no controle da infecção por A. reconditum.

\section{Agradecimentos}

Os autores dedicam o presente trabalho à memória do prof. Ennio Luz.

\section{Referências}

ALVES, L. C.; SILVA, L. V. A.; FAUSTINO, M. A. G.; McCALL, J. W.; SUPAKODERJ, P.; LABARTHE, N. W.; SANCHEZ, M.; CAIRES, O. Survey of canine heartworm in the city of Recife, Pernambuco, Brazil.
Memórias do Instituto Oswaldo Cruz, Rio de Janeiro, v. 94, n. 5, p. 587-590, 1999.

BARROS, M. T. G.; SANTOS, E. P.; ALMEIDA, M. A. O. Freqüência de microfilárias de Dipetalonema reconditum Grassi, 1890 em cães dos municípios de Salvador e Lauro de Freitas, Bahia. In: CONGRESSO INTERNACIONAL DE MEDICINA VETERINÁRIA EM LÍNGUA PORTUGUESA, 6., 1993, Salvador. Anais... Salvador: UFBA, 1993. p. 340.

BEAVER, P. C.; MEYER, E. A.; JARROL, E. L.; ROSENQUIST, R. C. Dipetalonema from the eye of a man in Oregon, USA. A case report. American Journal of Tropical Medicine \& Hygiene, USA, v. 29, n. 3, p. 369372, 1980.

BEAVER, P. C. Intraocular filariasis: a brief review. American Journal of Tropical Medicine \& Hygiene, USA, v. 40, n. 1, p. 40-45, 1989.

BRITO, A. C.; VILA-NOVA, M. C.; ROCHA, D. A. M.; COSTA, L. G.; ALMEIDA, W. A. P.; LOPES JUNIRO, R. R.; FONTES, G.; ROCHA, E. M. M.; REGIS, L. Prevalência de filariose canina causada por Dirofilaria immitis e Dipetalonema reconditum em Maceió, Alagoas, Brasil. Cadernos de Saúde Pública, Rio de Janeiro, v. 17, n. 6, p. 1497-1504, 2001.

CRINGOLI，G.; RINALDI，L.; VENEZIANO， V.; CAPELLI, G. A prevalence survey and risk analysis of filariosis in dogs from Mt. Vesuvius area of southern Italy. Veterinary Parasitology, Amsterdam, v. 102, n. 3, p. 243-252, 2001.

FERNANDES, C. G. N.; MOURA, S. T.; DIAS, A. R.; VIEIRA FILHO, W. S. Ocorrência de dirofilariose canina na região da Grande Cuiabá, estado de Mato Grosso Brasil. Brazilian Journal of Veterinary Research and Animal Science, São Paulo, v. 36, n. 5, p. 258-261, 1999.

FOROULIS, C. N.; KHALDI, L.; DESIMONAS, N.; KALAFATI, G. Pulmonary dirofilariasis mimicking lung tumor with chest wall and mediastinal invasion. The Journal of Thoracic and Cardiovascular Surgery, USA, v. 53, n. 3, p. 173-175, 2005.

GADAHI, J. A.; ARIJO, A. G.; ABUBAKAR, M.; JAVAID, S. B.; ARSHED, M. J. Prevalence of blood parasites in stray and pet dogs in Hyderabad area: comparative sensitivity of different diagnostic techniqes for the detection of microfilaria. Veterinary World, USA, v. 1, n. 8, p. 229-232, 2008.

GREENE, B. M.; OTTO, G. F.; GREENOUGH III, W. B. Circulating non-human microfilaria in a patient with Systemic Lupus Erythematosus. American Journal of Tropical Medicine and Higiene, USA, v. 27, n. 5, p. 905909, 1978. 
HASHEM, M.; BADAWY, A. I. I. Blood cellular and biochemical studies on filariasis of dogs. Research Journal of Animal Sciences, USA, v. 2, n. 5, p. 128-134, 2008.

HUYNH, T.; THEAN, J.; MAINI, I. Dipetalonema reconditum in the human eye. British Journal of Ophthalmology, London, v. 85, n. 11, p. 1384, 2001.

IBGE. Censo 2010. Disponível em: <http://www.ibge. gov.br/censo2010/index.php.>. Acesso em: 12 jan. 2011.

KNIGHT, D. H. Heartworm heart disease. Advances in Veterinary Sciences and Comparative Medicine, London, v. 21, p. 107-149, 1977.

. Heartworm infection. Veterinary Clinics of North America. Small Animals Practice, USA, v. 17, p. 1463-1518, 1987.

KNOTT, J. A method for making microfilarial surveys on day blood. Transactions of the Royal Society of Tropical Medicine and Higyene, USA, v. 23, n. 2, p. 191-196, 1939.

LEITE, L. C.; NAVARRO-SILVA, M. A; LUZ, E.; MOLINARI, H. P.; CIRIO, S. M.; MARINONI, L. P.; DINIZ, J. M. F.; LEITE, S. C.; LUNELLI, D.; SCALET, W. R. Prevalência de Dirofilaria immitis (Leidy, 1856) em cães do Canil Municipal de Guaratuba, Paraná, Brasil. Estudos de Biologia, Curitiba, v. 29, n. 66, p. 7379, 2007.

LINARDI, P. M. Biologia e epidemiologia das pulgas. Revista Brasileira de Parasitologia Veterinária, São Carlos, v. 13, p. 103-106, 2004. Suplemento 1.

NEWTON, W. L.; WRIGHT, W. H. The occurrence of a dog filariid other than Dirofilaria immitis in the U.S.A. Journal of Parasitology, Laurence, USA, v. 42, p. 246258, 1956.
RANI, P. A. M. A.; IRWIN, P. J.; GATNE, M.; COLEMAN, G. T.; TRAUB, R. J. Canine vectorborne diseases in India: a review of the literature and identification of existing knowledge gaps. Parasites \& Vectors, London, U.K., v. 3, n. 28, p. 1-7, 2010.

RANJBAR-BAHADORI, Sh.; LEKMATKHAH, A. A study on filariosis of stray dogs in Garmsar. Journal of Veterinary Research, USA, v. 62, n. 4, p. 73-76, 2007.

REIFUR, L.; THOMAZ-SOCCOL，V.; MONTIANIFERREIRA, F. Epidemiological aspects of filariosis on the coast of Paraná state, Brazil with emphasis on Dirofilaria immitis. Veterinary Parasitology, Michigan, USA, 122:273-286, 2004.

SABU, L.; DEVADA, K.; SUBRAMANIAN, H. Dirofilariosis in dogs \& humans in Kerala. Indian Journal of Medical Research, New Delhi, v. 121, p. 691693, 2005.

TOPARLAK, M.; GARGILI, A.; ULUTAS ESATGIL, M.; CETINKAYA, H. Canine filariosis around Istanbul, Turkey, employing Naphtol AS-TR Phosphatase. Acta Veterinaria Brunensis, Brno, Czech Republic, v. 74, n. 2, p. 233-236, 2005.

TORRES, F. D.; FIGUEREDO, L. A. Heterodoxus spiniger (Enderlein, 1909) on domestic dogs (Canis familiaris, L. 1758) from the city of Recife, Pernambuco State, Brazil. Brazilian Journal of Veterinary Research and Animal Science, São Paulo, v. 44, n. 2, p. 77-80, 2007.

WANG, L. C. Canine filarial infections in north Taiwan. Acta Tropica, Canada, v. 68, n. 1, p. 115-120, 1997.

ZAHLER, M.; GLASER, B.; GOTHE, R. Imported parasites in dogs: dirofilaria repens and dipetalonema reconditum. Tierarztl Prax, Germani, v. 25, n. 4, p. 388$392,1997$. 
